\title{
Multiscale analysis of geomorphological and geological features in high resolution digital elevation models using the wavelet transform
}

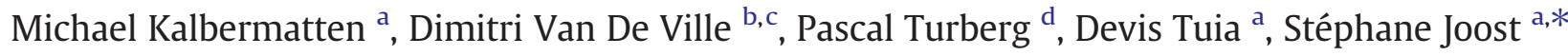 \\ a Laboratory of Geographical Information Systems (LASIG), Ecole Polytechnique Fédérale de Lausanne (EPFL), Station 18, CH-1015 Lausanne, Switzerland \\ ${ }^{\mathrm{b}}$ Medical Image Processing Lab, Ecole Polytechnique Fédérale de Lausanne (EPFL), Station 17, CH-1015 Lausanne, Switzerland \\ c University of Geneva, Rue Gabrielle-Perret-Gentil 4, CH-1211 Geneva, Switzerland \\ d Laboratory of Engineering and Environmental Geology (GEOLEP), Ecole Polytechnique Fédérale de Lausanne (EPFL), Station 18, CH-1015 Lausanne, Switzerland
}

\section{A R T I C L E I N F O}

\section{Article history:}

Received 16 July 2010

Received in revised form 6 September 2011

Accepted 24 September 2011

Available online 1 October 2011

\section{Keywords:}

Digital elevation model (DEM)

High resolution

Multiscale

Wavelets

Landslides

Jura

\begin{abstract}
A B S T R A C T
At the end of the 1990s the emergence of high resolution $(1 \mathrm{~m}$ ) digital elevation models (DEMs) settled the context of high precision geomorphological analysis. These new elevation models permitted to reveal structures that remained heretofore undetectable. Earth scientists henceforth benefit from a source of data with a textural detail that was never attained before. Despite its richness, this information must be treated efficiently to extract features helping geomorphologists to analyze the processes occurring at the surface. Such processes are complex, localized and naturally multiscale. Recently, space-frequential descriptors as wavelets have been proposed successfully for the analysis of DEMs. The wavelet transform is widely used in image processing since it allows us to decompose a signal into a weighted sum of atoms with joint space-frequency localization. Such a decomposition facilitates a coherent navigation from scale to scale, but also permits to detect heretofore undiscerned phenomena at different scales. This is appealing in geomorphology, where structural components, related to a given phenomenon, are well determined in these sub-spaces specific to the scale continuum. In this paper, we propose a filtering procedure of the wavelet decomposition as an approach for the analysis of geomorphological multiscale structures. This filtering procedure enhances the high pass information contained at each scale. The proposed bottom-up approach is applied here to a case study to detect geomorphological structural elements in a valley of the Swiss Jura. It demonstrates that the proposed filtering procedure is an efficient tool for geomorphological multiscale generalization.
\end{abstract}

(c) 2011 Elsevier B.V. All rights reserved.

\section{Introduction}

Digital elevation models (DEMs) have fundamentally changed the way we perceive elevation information. In the late 1990s, the emergence of high resolution $(\sim 1 \mathrm{~m})$ elevation data allowed the exploration of our environment and its morphology with an unprecedented level of detail, making new applications possible (e.g. the study of micro-faults on cliffs). The visual analysis of a shaded DEM efficiently supports the detection of a great amount of features at various scales. Nowadays, earth-science experts such as geologists and geomorphologists use high resolution DEMs to visually assess geomorphological features. Thanks to the finer description offered by this new and rich source of information, the study of visual perception, i.e. the visible phenomena or relevant structures, has evolved considerably. Indeed, it is now possible for instance to visually analyze a hillside and its details (elements of only a few meters).

\footnotetext{
* Corresponding author. Tel.: +4121 6935782; fax: +41216935790. E-mail address: stephane.joost@epfl.ch (S. Joost).
}

Since geological phenomena are composed of different nested topographical features, a multiscale approach is essential in geomorphological analysis. Klinkenberg (1992) suggested that a phenomenon fits over scales and that its features are nested in discrete scale intervals, leading to a strong correlation between features and phenomena (Mark and Aronson, 1984). In human vision, the neural network is able to distinguish specific features in relation to a corresponding scale (Marr, 1982), as well as to carry out a multiresolution analysis. As suggested by Marr (1982), our visual system is probably linked to tuned cells or, in other words, it has specific frequency intervals to which it is sensitive. Therefore, computer systems and the visual representations we make of processed data should reflect this multiscale nature. Nevertheless, in most current systems, information is perceived like a static image. There are two ways to interpret information in an image: either we know what the image contains and we focus on retrieving this information using the most appropriate technique; or we do not know what the image contains and we use a more general method to identify and differentiate relevant content in the data. The latter applies to DEM analysis. Often, a given DEM contains specific information like a geological phenomenon, but the identification of its components is not straightforward. Consequently, topographical 
features have to be classified according to their representative scale. In other words, it is necessary to find the best correlation between a certain level of generalization (of the DEM) and the scale of a particular feature of the topography. This process is called a multiscale analysis of structural topographical features (Marceau and Hay, 1999).

High resolution DEMs offer the base conditions to perform such an analysis. High resolution describes very fine structural levels that stem from different processes. For example, a micro-fold may result from a landslide or from erosion. Thus, the imprint of this feature will not be contained in the same spatial context regarding its relation to coarser features. Although high resolution provides a much better visual rendering of the territory and of its structures, the relations between topographical structures and formations are more complicated. Hence they represent a new challenge for quantitative geomorphology and geomorphometry.

A first attempt to address this challenge was carried out through the development of form indicators, also known as geomorphometry indicators (e.g., Wood, 1996). Geomorphometric indicators are spatial features that can be extracted from a DEM, such as slope, aspect, and curvature. These indicators are geometric because they are computed using the adjustment of a mathematical surface on elevation models. The detection of geomorphological features using this type of indicators (as well as hydro-morphological indicators such as the wetness index, watersheds, and streams) is complicated because such indices are dedicated to local scale analysis only. Moreover, in high resolution DEMs, features are nested one into the other, making the interpretation of indicators difficult. This is a computational scale problem (see Lassueur et al., 2006, and references therein) also observed in the use of geomorphometric indicators for the prediction of environmental parameters such as wind speed (Foresti et al., 2011) and orographic precipitation (Foresti and Pozdnoukhov, in press). Wilson and Gallant (2000) showed that the characterization of landscape processes and features based on one specific scale is far too simple to model our environment. In recent years, multiresolution analysis tools based on a generalization of Evans' (1972) geomorphometric indicators have been developed (Wood, 1996). These tools provide multiple results for one indicator at multiple scales. There is no feature extraction, but rather a multiscale/multiresolution topographical analysis and the extraction of a geometric network. These methods rely essentially on a geometrical analysis, while Jordan and colleagues combined geomorphometric indicators and digital image processing techniques (including edge detectors, histogram slicing, and gradient filters) to detect tectonic faults with DEMs (Jordan et al., 2005; Jordan, 2007).

To enable the detection of a phenomenon and its underlying features, it is necessary to identify the specific scales at which significant features and their intrinsic relations emerge. A way to move toward the extraction of geomorphological indicators at different scales is to consider DEMs in the frequency domain. Indeed, our environment is composed of frequency information characterizing either the spatial domain (as it is considered in this paper) or the temporal domain (e.g. earthquakes or mass movements). A limitation is that almost no natural phenomenon related to Earth science is stationary and homogeneous, and this made many studies fail. Real phenomena consist of a nesting of processes and structural elements, which are interdependent, have various scales, and interact in the natural system. This means that the size and shape of every structure depends on the phenomenon it belongs to, but also on the functional scale of the system.

If we link a basic shape, which can be represented by a function, to space-frequency analysis, we might develop a rigorous and exhaustive multiscale structural analysis. Moreover, the best representation of our function at a specific scale level would illustrate which types of structures arise at the same level. De Boer (1992) suggested that scale should be used as an analytical framework to reduce the high frequencies caused by finer scale levels. The idea is to focus on the functionalities and properties of a DEM at a certain scale. If we consider frequency analysis, the Fourier transform cannot be used to perform this complex operation due to the stationarity assumption. But the wavelet transform we consider in this work is able to combine spatial and spectral localization. It also has localization and compact support properties to fulfill our needs. "Compact support" means that the function associated with the transform is null out of a given spatial bound.

The use of wavelet decomposition with DEMs is not novel, but previously published works mainly dealt with problems of compression of the DEM (Ferretti et al., 1999; Creusere, 2001; Ottoson, 2001). In parallel, there has been a new current of research considering the analysis of elevation models using wavelet decomposition (Datcu et al., 1996; Gallant and Hutchinson, 1996; Pike, 2000; Mahler, 2001; Martinoni, 2002; Amgaa, 2003; Bjorke and Nilsen, 2003; Jordan and Schott, 2005; Lashermes et al., 2007). This work falls within the scope of the latter.

With respect to feature extraction, Grewe and Brooks (1997) and Amgaa (2003) showed that the considered feature has to be of high gradient limit. Indeed, as most of geomorphological features are nested one into the other, their delimitation is a difficult task. This is probably because autocorrelation is higher than in usual images. Vu and Tokunaga (2002) and Amgaa (2003) applied wavelets to the extraction and segmentation of surface models containing building information. Here, wavelets are efficient because of the sharp and clean building limits. Datcu et al. (1996) estimated the fractal dimension of DEMs using features extracted from the wavelet decomposition in order to segment the roughness of the DEM. Lashermes et al. (2007) also used wavelets to extract curvatures and slope/direction changes in DEMs in order to identify channel networks defining the transition between hillslopes and valleys.

Related to DEM filtering, Mahler (2001) and Martinoni (2002) filtered wavelet coefficients to generate filtered versions of the original DEM. They also gave some hints on how to isolate specific scale information. Indeed, since the deployed wavelets may not be symmetric (Daubechies) or smooth enough (Haar) for terrain representation, and since their shape may not well fit natural structures, the generalization and filtering process easily induces artifacts. Bjorke and Nilsen (2003) also applied a similar approach using the Haar wavelet.

Finally, Mahler (2001) filtered high resolution DEMs according to the desired scale level using wavelets. Still, his major interest resided in the filtered DEM and not in the analysis of the residuals, i.e. the noise removed with a multiscale filter.

In this paper, we study the possibility to define a framework involving scale-driven processes and the delimitation of scale intervals. We cannot have any a priori knowledge of the best partition and representation of the scale spaces. An exploratory approach is therefore necessary to apprehend them, as well as to understand the link between structures, processes and phenomena. We define these spaces by interpreting and analyzing a certain reality by means of multiscale representation. In interpreting these spaces, we try to reconstruct the reality of the processes that affect a topographical phenomenon, not only regarding their formal definition but also in the context of the structural nesting they are fitted into.

We analyzed the scale of topographical features according to a novel and explicit discretization of the continuous scale, computed using the wavelet transform, but filtered by successive elimination of low-pass information contained in the DEM. Then, using the inverse wavelet transform, we reconstructed a high resolution image containing only high-pass information for a series of scales. We applied this methodology to a DEM covering a recent landslide located in Travers in the Canton de Neuchâtel (Switzerland). The results provided a good correspondence with a standard geomorphological analysis carried out in the field: characterized features were compared with the different high-pass images computed for identification and for the attribution to a specific scale interval. 


\section{Generalization process using wavelets}

The wavelet transform is based on two intricately linked functions: the scaling function, which could be a B-spline base of third degree as in our case (Unser, 1999), and the wavelet function. The wavelet coefficients can be obtained with an efficient filter bank algorithm and are equivalent to inner products of the signal with shifted and dilated versions of the wavelet. The two functions are detailed in the next sections. A complete introduction to wavelets and the underlying transform may be found in the tutorial section of http:// www.wavelet.org (accessed September 2, 2011).

Here we use the two-dimensional wavelet transform, which applies the conventional one-dimensional transform sequentially along each dimension. The transform results in $2^{i}$ resolution images (where $i$ is the level of decomposition, $i=0,1 \ldots 8$, with 0 being the original DEM). An example is given in Fig. 1. Due to dyadic subsampling, the resolution between succeeding scales is multiplied by a factor of two and three different wavelets are obtained at each scale (i.e., combinations of horizontal wavelet with vertical scaling function and vice versa, and horizontal with vertical wavelet). The $c_{i+1}[2 k]$ coefficients correspond to the downsampled low-pass image of the original DEM $c_{i}[k]$, while $d_{v, i+1}[2 k], d_{d, i+1}[2 k]$ and $d_{h, i+1}[2 k]$ are the highpass coefficients of the $(i+1)^{\text {th }}$ decomposition level $(v$ for vertical high-pass coefficients, $h$ for horizontal high-pass coefficients and $d$ for diagonal high-pass coefficients). The application of the wavelet transform (WT) on DEMs and its basic minimalistic parameterization enable the replication of the methodology on other DEMs.

\subsection{B-spline interpolation}

Mathematically, WT used here is linked to an interpolation using a B-spline basis. The basis of degree 0 is defined by Dierkcx (1993):

$\beta^{0}(x)=\left\{\begin{array}{l}1, \text { if }-1 / 2 \leq x<1 / 2 \\ 0, \text { otherwise }\end{array}\right.$

For all degree $n$, this interpolation function can be defined through the $n+1$ convolution of the basis function with degree 0 :

$\beta^{n}(x)=\underbrace{\beta^{0} * \beta^{0} * \ldots * \beta^{0}}_{n+1 \text { times }}$.

Schoenberg (1946) defined a spline as any linear combination of shifted B-splines:

$s(x)=\sum_{k \in \mathbb{Z}} c(k) \beta^{n}(x-k)=\left(c \times b_{1}^{n}\right)(k)$

where $s(x)$ is a signal and $c(k)$ are the B-spline coefficients.

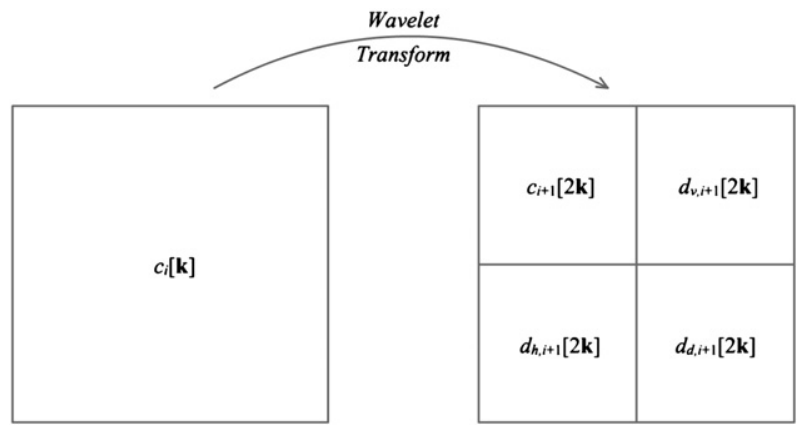

Fig. 1. Conceptual framework of the wavelet transform. The $c_{i}[k]$ coefficients are downsampled into four images $c_{i+1}[2 k], d_{(v, i+1)}[2 k], d_{(d, i+1)}[2 k]$ and $d_{(h, i+1)}[2 k]$, adapted from Mallat (2000). The $c_{i+1}[2 k]$ coefficients represent the downsampled DEM. The three other resulting images are the high-pass information regarding respectively the vertical, the diagonal and the horizontal directions. They represent all information which was not transferred from level $i$ to level $i+1$.
This convolution in the spatial domain can be written as a multiplication in the Fourier domain:

$S\left(e^{j \omega}\right)=C\left(e^{j \omega}\right) \cdot B\left(e^{j \omega}\right)$

where $e^{j \omega}$ is the complex grain (time-frequency or position-frequency in our case) of the signal and $\omega$ is the angular frequency in the Fourier domain.

The $B\left(e^{j \omega}\right)$ coefficients can be calculated using the autocorrelation filter of a B-spline of degree $n$ (Unser and Blu, 1999; Blu and Unser, 2000):

$B\left(e^{j \omega}\right)=\sum_{k=-\infty}^{+\infty} \hat{\beta}^{n}(\omega+2 \pi k)=\sum_{k=-\infty}^{+\infty}\left|\hat{\beta}^{\frac{n-1}{4}}(\omega+2 \pi k)\right|^{2}=A^{\frac{n-1}{2}}\left(e^{j \omega}\right)$

where $\hat{\beta}^{n}$ is the impulse response of $\beta$ in the Fourier domain.

\subsection{Wavelet transform}

Mallat (2000) defined the continuous wavelet transform $\psi \in L^{2}(\mathbb{R})$ by using a function with zero mean:

$\int_{-\infty}^{\infty} \psi(x) d x=0$

If the wavelet is dilated by a factor $s$ and translated by $u$, it gives:

$\psi_{u, s}(x)=\frac{1}{\sqrt{s}} \psi\left(\frac{x-u}{s}\right)$.

Thus the wavelet transform $W f$ of $f \in L^{2}(\mathbb{R})$ at position $x$ and scale $s$ is:

$W f(u, s)=\left\langle f, \psi_{u, s}\right\rangle=\int_{-\infty}^{\infty} f(x) \frac{1}{\sqrt{s}} \psi^{*}\left(\frac{x-u}{s}\right) d x$

where $\psi^{*}$ is the complex conjugate of the wavelet $\psi$.

This transform can be rewritten as a convolution:

$W f(u, s)=\left\langle f, \psi_{u, s}\right\rangle=f \times \bar{\psi}_{s}(u)$

where $\bar{\psi}_{s}(x)=\frac{1}{\sqrt{s}} \psi^{*}\left(\frac{-\chi}{s}\right)$ is the scaled wavelet at position $u$.

Mallat (2000) proved that if a scaling function $\varphi(x)$ satisfies three validity conditions, a corresponding wavelet $\psi(x)$ exists and is a linear combination of translated versions of the scaling function at the finer resolution (Unser and Blu, 2003):

$\psi\left(\frac{x}{2}\right)=\sum_{k \in \mathbb{Z}} g(k) \varphi(x-k)$

where $g(k)$ is the wavelet (high-pass) filter in the spatial domain.

The refinement condition enables us to define:

$\varphi\left(\frac{x}{2}\right)=\sum_{k \in \mathbb{Z}} h(k) \varphi(x-k)$

where $h(k)$ is the low-pass complement of the wavelet filter in the spatial domain.

For computational purposes, it is faster to process the signal in the Fourier domain (identified hereafter with "^" symbol): $2 \hat{\varphi}(2 \omega)=$ $\hat{h}(\omega) \hat{\varphi}(\omega)$ and finally:

$H\left(e^{j \omega}\right)=2 \frac{\hat{\varphi}(2 \omega)}{\hat{\varphi}(\omega)}$.

The B-spline basis we defined earlier obeys to the three conditions of the wavelet basis function. We may use it $\left(\varphi=\beta^{n}\right)$ and define the two quadrature filters needed by the wavelet transform. According to Mallat 


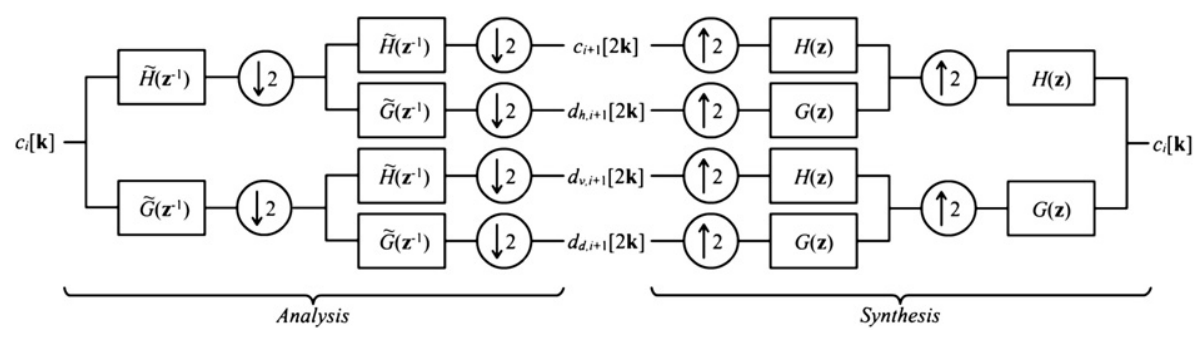

Fig. 2. Mallat's analysis and synthesis quadrature filter bank (Mallat, 2000). First the columns are filtered and downsampled; then the rows are filtered and downsampled.

(2000), the transform can be described as in Fig. 2. In this paper, we use a B-spline basis of third degree as scaling function, thus $n=3$.

\subsection{Inverse wavelet transform}

As mentioned in the previous section, the wavelet transform consists of the definition of two analysis filters depending on the used base (or wavelet) function. Vetterli (1986) defined the necessary conditions for a perfect reconstruction of the original signal (the DEM in our case). Our analysis function respects those conditions and the synthesis filters can be defined as follows:

$\tilde{H}\left(e^{j \omega}\right) H\left(e^{j \omega}\right)+\tilde{G}\left(e^{j \omega}\right) G\left(e^{j \omega}\right)=2$

$\tilde{H}\left(e^{j(\omega+\pi)}\right) H\left(e^{j \omega}\right)+\tilde{G}\left(e^{j(\omega+\pi)}\right) G\left(e^{j \omega}\right)=0$

where $\tilde{H}\left(e^{j \omega}\right)$ is the dual version of $H\left(e^{j \omega}\right)$ and $\tilde{G}\left(e^{j \omega}\right)$ is the dual version of $G\left(e^{j \omega}\right)$.

The first condition is the distortion-free condition and the second one the anti-aliasing condition.

On these bases, we are able to perform an analysis (decomposition) of the signal and its perfect synthesis (reconstruction) over the positionfrequency domain.

\subsection{Proposed filtering procedure}

From one level to the other, all elements that are not conserved in a DEM (low-pass image) necessarily lie within high-pass images (detail coefficients). For each decomposition level, it is possible to replace the DEM matrix with a zero matrix and then to rebuild the original image, thus to rebuild specific subbands of the wavelet transform.

The key process of such a filtering is a hierarchical classification of the information depending on scale, i.e. the consolidation of structural elements ranging from the finest resolution to the resolution of the decomposition level. Consider the decomposition reported in Fig. 3: in this case, the original DEM has a 1-m resolution. If the data are processed to the second decomposition level, the resulting images (or DEM) will show a 4-m resolution. At this resolution, the approach schematized in Fig. 3 is therefore expected to detect structural elements ranging approximately from 1 to $4 \mathrm{~m}$ radii. In the next sections, the complete process is detailed for the detection of geomorphological structural elements generated by a landslide that occurred in a valley of the Swiss Jura in 2007.

\section{Case study: the Travers landslide in the Swiss Jura}

The valley in which the landslide occurred is part of the Jura, a mountainous region located between Franche-Comté in France and the region of Basel in Switzerland. The local side slope is composed of moraine and limestone. The upper part of the slope is interfered with earlier fallen rocks. The landslide occurred in April 2007 on a moderate slope after a heavy rain and snow-melting period. In order to interpolate a DEM, LiDAR (Light Detection And Ranging) data were acquired ten days after the landslide. As shown on the in situ descriptive structure map (Krähenbühl, 2007) (Fig. 4), the landslide (of type "earth flowlandslide") is composed of a small scarp zone (about $2500 \mathrm{~m}^{2}$ ) followed by a de-structured accumulation zone (about $5700 \mathrm{~m}^{2}$ ) going from the old road path to about $20 \mathrm{~m}$ under the old road waste. In its upper part (south-east), it shows a major scarp zone made of moraine, a matrix of clay and sandy muddy alterites of the bedrock molasses. The end of the accumulation zone is a differential settling of the material. The water resurgences that appeared afterwards did not permit a definitive scarp zone stabilization. Five months after the event (August), material was still slightly moving. The landslide is covering a 32,000 $\mathrm{m}^{2}$ area, mainly used for agriculture and pasture.

Materials slid along a fracture surface parallel to the slope (translational landslide to the level of the roof of the molasse) according to two different modes. First, the displaced material, rich in sandy muddy saturated material, was liquefied and produced a flow, which now occupies the upper central and eastern parts of the accumulation zone (see the in situ descriptive structure map reported in Fig. 4). Second, in the continuity of the first movement mode, the morainic displaced material and its pedological coverage moved in a more ductile way and depleted on the accumulation zone. The resulting folds are clearly distinguishable on the north-west part of the accumulation zone (Fig. 4). These folds show a homogeneous distribution according to their frequencies. Indeed, it is a frequency imbrication of terrain folds. The lowest has a wavelength of approximately $40 \mathrm{~m}$ with a shorter wavelength (2-4 m) superposition.

Four subterranean water resurgences appeared after the landslide occurred: three in the scarp zone and one in the middle of the accumulation zone. This happened because the displaced and bedrock materials were saturated at the time of the event. This material induced the evacuation of subterranean water under pressure, and several wetlands as well as ponds appeared in the accumulation zone. The three scarp zone resurgences were drained (on surface) to a spillway in order to reconstruct a new road, stabilized with tree trunk stilts. The accumulation zone (compressed material) starts at the former road path and is composed of a series of big folds, in which undulating micro-structural elements can be seen.

We undertook a wavelet analysis of the DEM in order to assess the nesting of typical structural elements and to provide a multiscale view of these to geomorphologists. The elements were computed using different enhancement filters ( step F in Fig. 3), thus giving multiple results and views of structural nesting at each scale. The filters enable to enhance high-pass information of specific decomposition levels. Finally, the computed DEMs were compared with a visual delimitation of landslide structural elements elaborated by an expert geologist.

\section{Results}

Two distinct analyses are reported. The first is a global analysis of the landslide zone using the filtered coefficients. The second is a specific landslide analysis of the WT filtering effect. 

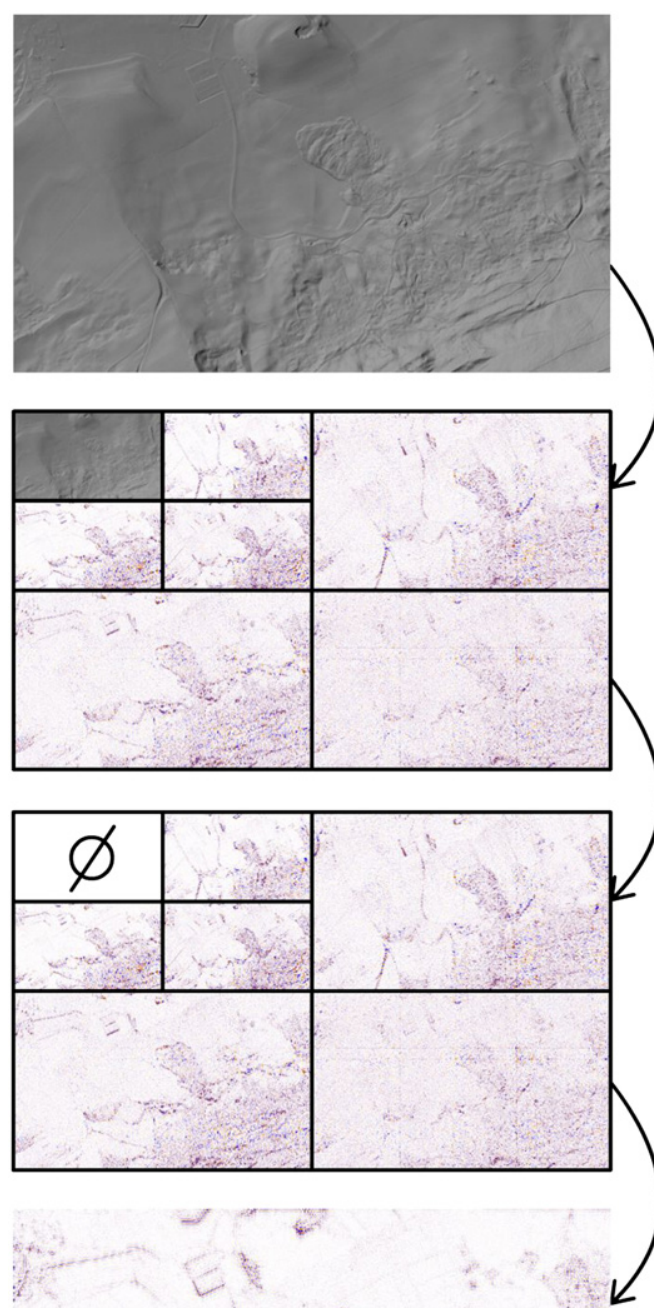

WT: Inverse wavelet transform; it is applied to the coefficients (except for the low-pass). The result is an image containing all the high-pass information considering the chosen decomposition level (the second one in this case).

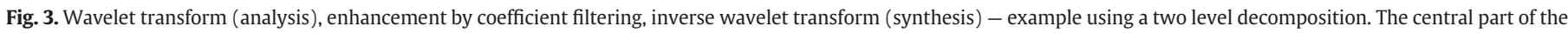
image reported illustrates the landslide studied in the experimental sections.

\subsection{Landslide structural elements}

A zonal delimitation was undertaken by a geologist using the aerial photography, terrain knowledge and observations. This analysis resulted in the definition of seven different zones (Fig. 5):

- Zone I, corresponding to the plastic and solid deformations at the downstream of the landslide, laterally surrounded by more destructive zones (zones II and V).

- Zone II, where the debris flow from the scarp zone was deposited, mainly liquefied by the water resurgences.

- Zone III, the main scarp zone, smaller than the other zones, but supplied all the deposit material.

- Zone IV, transition zone between the liquefied material of zone II and the meadow surrounding the landslide, composed of several folds due to differential displacement of material.

- Zone V, transition between the side of zone I and the meadow, like zone IV.
- Zone VI, could be linked to zone I, because it is not from the liquefaction process, but the material sustained a plastic settlement.

- Zone VII, small zone influenced by the nearby material displacement, showing some minor folds and discontinuities.

Fig. 6 illustrates the wavelet high-pass coefficients reconstructed with the proposed method. The coefficients of these images were normalized in the interval $[-100 ; 100]$ and their color saturation level was set to $[-20 ; 20]$. Positive coefficients are shown in red tones and indicate terrain convexity. Negative coefficients are shown in blue tones and indicate terrain concavity.

Looking at the size of the zones, there is no chance for them to appear in the four first decomposition levels. In Fig. 6, the end of the accumulation (south-west of zone I) is clearly visible because the terrain heterogeneity decreases. Zones II and III show similar behaviors. At the seventh and eighth levels, some of the defined zones do not respect the delimitation of the wavelet spatial recognition. For example, zone IV is not as rectilinear as drawn by the expert, 


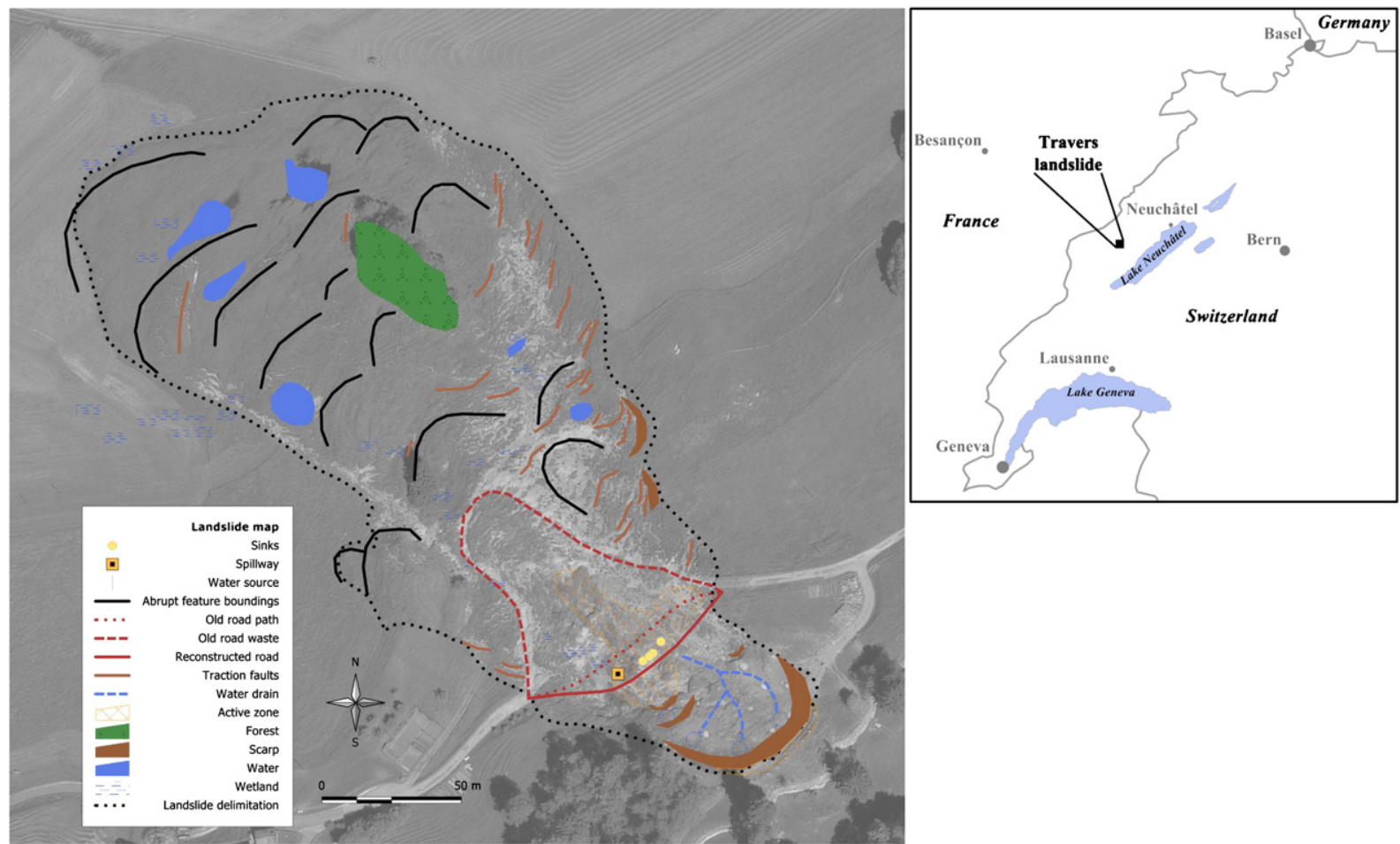

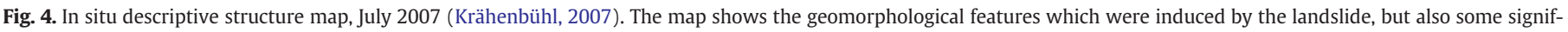

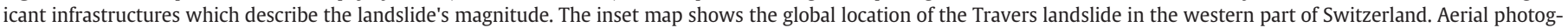
raphy (c) SITN.

but it intersects a depression (blue on the images), which is a continuity of zone II. Zone V is the only one which cannot be identified (even partially). Indeed, on the aerial photography it can hardly be recognized as an entity.

\subsection{Landslide detailed elements}

Based on the observations of the previous section, we can now identify three areas in order to make a more detailed analysis of their

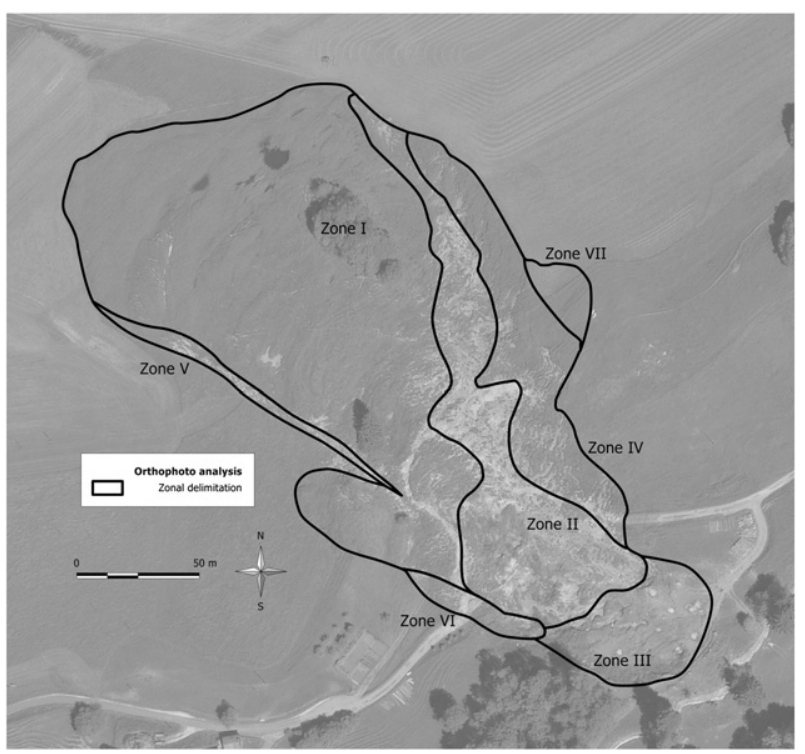

Fig. 5. Visual delimitation of structural elements using aerial photography. Zone I corresponds to plastic and solid deformations (no direct deposit). Zone II is the deposit zone of material which flowed down from the scarp zone. Zone III is the scarp zone. Zones IV to VII are lateral and transition zones of the landslide. They were affected by some deformations or some secondary deposits. Aerial photography (c) SITN. subband components. They spatially cover all main structural elements of the landslide (scarp zone, settlement in accumulation zone and fluid accumulation zone) and can be described as follows (Fig. 7):

- Zone A $\left(5360 \mathrm{~m}^{2}\right)$ is located in the toe of the landslide, thus in the settlement of the accumulation zone. It mainly contains wave structures due to the plastic behavior of the phenomenon. The settlement is inherent to the uphill mass movement.

- Zone B $\left(2720 \mathrm{~m}^{2}\right)$ is the continuity of the material flow which occurred downhill from the scarp zone. The material consists mainly of clay and sandy liquefied molasse. It is therefore relevant to the mass movement of fluid material of the landslide.

- Zone C $\left(1810 \mathrm{~m}^{2}\right)$ is simply the whole scarp zone. It is the smallest of the three zones. Because this area was reshaped and reorganized by the authorities to ensure the drainage of surface water to the spillway, the structures composing it are very sharp. There are three surface channels which intersect at the bottom of the scarp zone toward the spillway.

Identification of landforms is never unique and dissimilarities may appear when comparing results of different experts. Rather than probing a geological expert, the idea here is to study and visualize the scale imbrication structures, as well as their impact on the phenomena. Moreover, using smaller areas would certainly provide results, but because of their size, the structural elements inside would be hard to describe from a geological point of view.

\subsubsection{Zone A}

The overlay between zone A and the high-pass reconstructed images is shown in Fig. 8. In the first decomposition levels (1 to 3), it is noteworthy that the linear structure types are bent in the direction of the mass movement (north-west). Moreover, there is a low roughness area between the north-west and the south-west of this zone, as if the structures had lower amplitude in the center of the zone. However, this effect disappears when going to decomposition level 4. Indeed, the enhancement of the fine-resolution subbands 


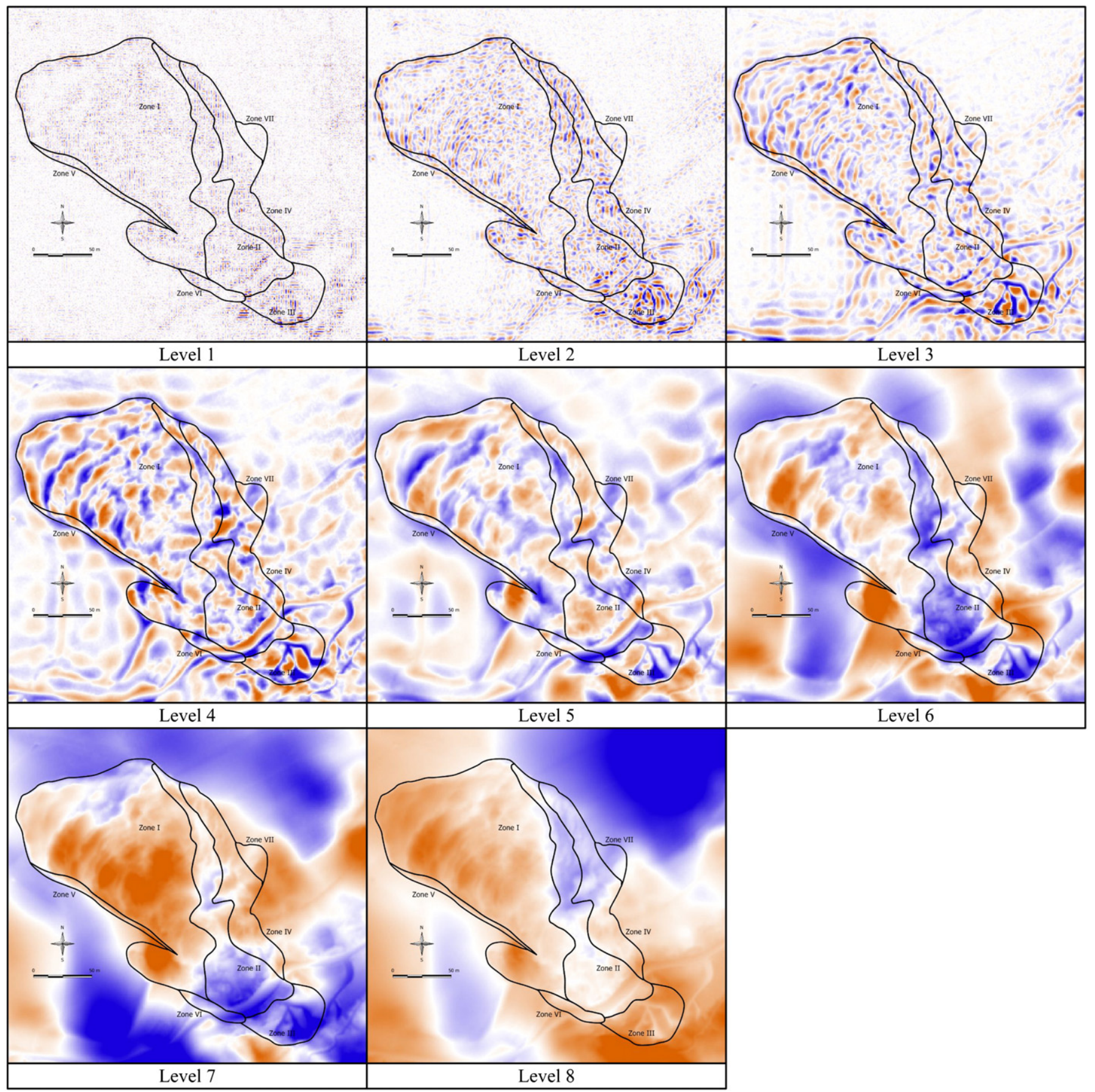

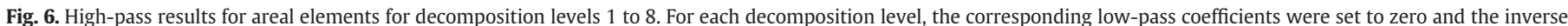
transform was applied in order to represent level specific information.

highlights the micro-structures contained in the zone. At decomposition level 4 (see Fig. 8), the separation of the zone into two distinct convex areas is visible. The two areas are separated by a sharp concavity highlighted by the amplitude of the coefficient (i.e. the saturation of the blue color) visible on the image. As for these two subzones, they are composed of micro-structures of lower magnitude.

The separation of zone A into two sub-zones is visually effective in levels 5 and 6 . It clearly identifies the landslide toe and another uphill area (the two red zones in zone A). There is a terrace-like effect where the toe is disturbed by micro-folds. Schematically, zone A represents the toe and a secondary settlement area (see Fig. 9). The latter is less perturbed by micro-folds and this is probably due to the differential settlement. Indeed, the forces induced by the weight of the materials are extreme at the bottom of the mass movement.

Finally, decomposition levels 7 and 8 in Fig. 8 clearly show that the inherent dimensions of the represented features are larger than the considered zone, and therefore the related scale spaces cover larger features than those composing zone A. This illustrates the spatial boundaries of this zone. Moreover, level 6 has a resolution of $64 \mathrm{~m}^{2}$ $\left(2^{6}\right)$ and is related to features of about $100-150 \mathrm{~m}$ (perimeter); this level is the last to represent features related to this zone. This does not mean that coarser features did not interfere with the process, but only that the biggest physical features inside zone $\mathrm{A}$ have a size of about $100-150 \mathrm{~m}$. 


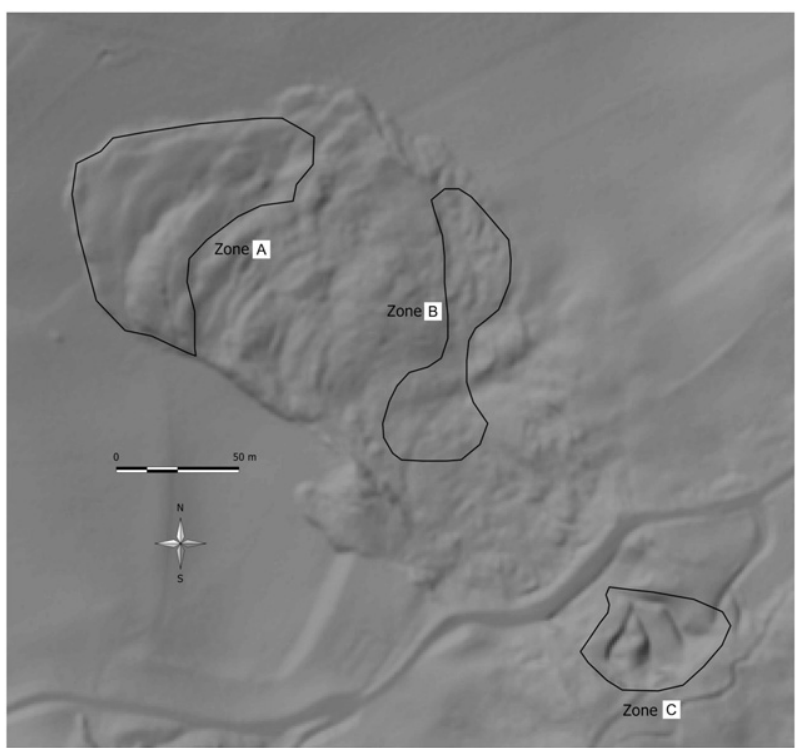

Fig. 7. Visual delimitation of structural elements (zones A, B and C) in the 1-m resolution shaded DEM. DEM @ SITN.

\subsubsection{Zone B}

The overlay between zone B and the high-pass reconstructed images is shown in Fig. 8. Structural elements in the North of the zone show the preferential direction of mass movements in the first decomposition levels (1 to 3 ). The second level distinctly shows the fluid flows to which this area was subjected by the fact that the linear structures are oriented from the South to the North. From level 4, the linear structures tend to disappear for the benefit of areal structural elements. The defined zone seems less consistent compared with zone A: it is more difficult to recognize this zone through the visual analysis of the images. Decomposition levels 5 and 6 show a subdivision of the zone into two separate areas. The first is a sort of bump of rather low convexity in the southern part of the zone and the second is a concave hollow area in the North. Levels 7 and 8 do not provide any additional information about the structural elements.

\subsubsection{Zone C}

Zone $\mathrm{C}$ is shown in Fig. 10. In this area the first two levels are different because of the structural configuration of the scarp zone (anthropogenic alteration). Level 1 shows the intense reaction of the wavelets to the drainage canals dug by the authorities. High magnitude oriented structures in two dimensions ( $\mathrm{x}$ and $\mathrm{y}$ axis) are visible. At level 2, it is possible to guess the reconstructed shape of the canals with very sharp shapes corresponding to the wavelets of the second frequency space. The next two levels ( 3 and 4 ) do not provide any extra information. At the fifth level, the location of the spillway is obvious. However, there is no additional useful information. At level six, the scarp zone is aggregated with the rest of the flow zone, including the new road (north-west area). Finally, at higher levels the scarp zone is completely absorbed by hillside phenomena, whose scale exceeds by far the size and structure of zone C.

\subsection{Characterization of scale-dependent processes}

To sum up the short description of zones presented in the previous section, we observed that high-pass images improve human visual capabilities by applying selective filtering to the raw data. Enhancement of specific structural information improves our comprehension of the phenomenon. The analyses performed and interpretations of the wavelet transform results are purely visual and contextual. This means that they are determined according to our analytical capabilities, without recourse to any deterministic or stochastic indicator. However, the wavelet transform and the associated enhancement filters provide interesting results necessary to carry out a morphological interpretation of geological phenomena. The structural analysis shows that the high-pass images yield a lot of indications regarding geomorphological elements contained in DEMs. The multiscale approach is thus able to produce a nested vision of morphological features.

In the following, we tried to characterize the observed elements into four types of phenomena that span scale (Fig. 11). The delimited spatial extent refers to the different partitions of the sizes of the features we were able to visually identify. Starting with the micro-scale and moving toward the macro-scale, the structural hierarchy throughout the scale space is a function of the DEM resolution and can be organized as follows:

- The domain of terrain roughness analysis is defined from levels 1 to 3 .

- The domain of internal individual structures is defined from levels 3 to 5 . This domain refers to isolated structures inside the landslide like folds, faults or the hydrological network.

- The domain of internal general structures is defined from levels 5 to 8. This domain illustrates the different zonal partitions within the landslide. It is defined by zones within which the different elements of the second domain are grouped.

- The domain of local geological structures is defined from levels 8 to the upper ones. Geological structures are those of foothills and local geomorphological features of a bigger size than the features composing the landslide.

This partition is a first attempt to highlight the scale spaces of interest for the Travers DEM and it should be admitted with caution. Nonetheless, it provides an overview of the structural organization and constituted a helpful support for a multiscale characterization of the different structural elements.

The discretization of spatial scales illustrates which structural elements can be found in which partition. Moreover, its wavelet decomposition levels cover less and less frequencies as we subdivide, but their coefficients show gradually higher magnitudes, demonstrating that the amplitude of features increases along with the decomposition level. It also shows that a geomorphological phenomenon consists of structural elements observable at different scales. Throughout this analysis, it must be emphasized that the space partition covering each of the four domains defined spans about two wavelet decomposition levels.

\section{Discussion}

Wavelets and the underlying transform are highly relevant analytical tools for multiresolution analysis. The dyadic subsampling makes it possible to clearly pre-define the scale partition. The spectral localized investigation of the wavelet transform identifies frequency properties of terrain features while non-localized methods like the Fourier transform cannot be used in this case (Jordan and Schott, 2005). Indeed, if we suppress or filter some frequencies in the Fourier domain, we do not exactly know what the implications in the spatial domain will be. As it has been observed experimentally, wavelets are ideal tools to get rid of this limitation. The filter bank simplicity allows us i) to create multiple frequency subspaces in the spatial domain, and ii) to analyze specific and combined scale intervals by subband suppression. Furthermore, the wavelet transform is not a geometrical analysis like those conducted by usual geomorphometric indicators. It generates scale dependent coefficients with a given positive or negative magnitude. However, the high-pass information contained in the single levels of decomposition lacks a context and a single scale image is therefore difficult to interpret, as observed in the visual analysis reported. This is due to the fact that the frequency domain is partitioned into different levels that individually are not informative, since both the 


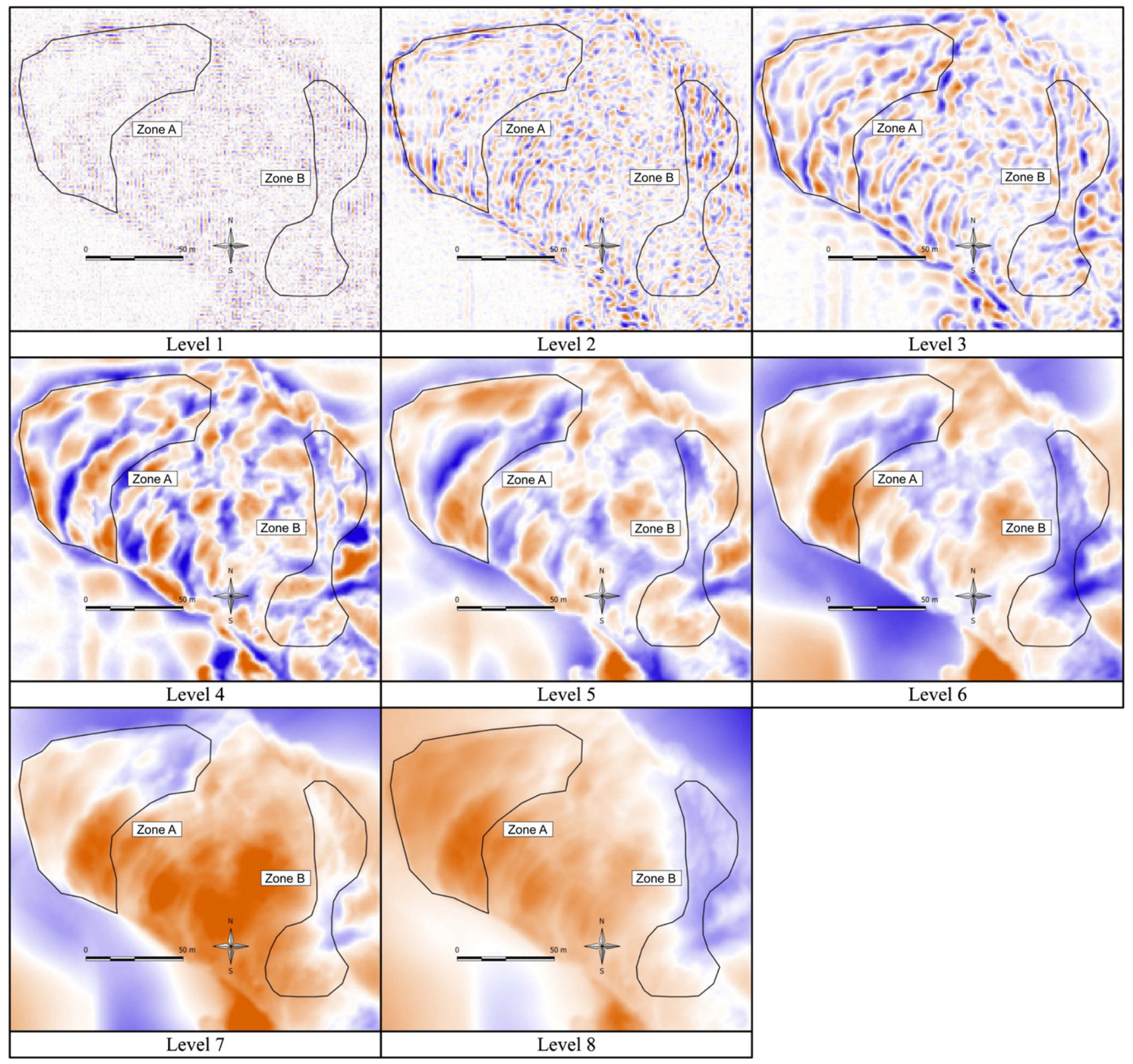

Fig. 8. High-pass results for zones A and B for decomposition levels 1 to 8 .

nesting of structural information and the geomorphological context are lost. This constitutes the main limitation of the analysis. To avoid such a loss of context, we used a combination of the high-pass information contained in the subsequent levels to describe scale-dependent

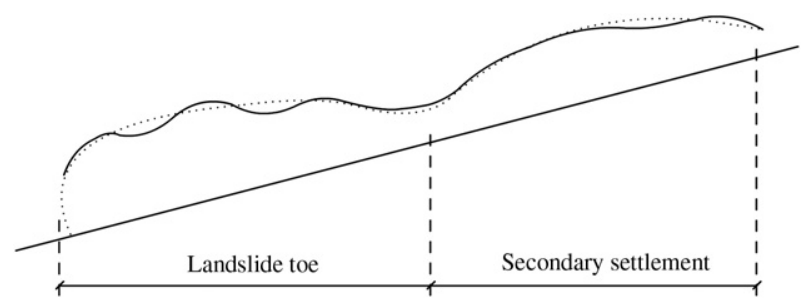

Fig. 9. Schematic profile of the toe structures. The dashed line shows the global profile tendency, whereas the solid line shows the local structures. features. The finer subbands provide context to scale, so that the nesting of structural information is maintained and visualizable.

The filtering of high-pass coefficient levels performed is a way to exaggerate specific scale interval information. Nonetheless, this operation can only be undertaken once we know which structural elements are represented at which levels. There is clearly a lack of standard specification of the relevant scale intervals of the wavelet decomposition. This need pushed us to define the domains shown in Fig. 11. These scale intervals provide reference ranges to identify various structural types which may be observed in reality. To do so, it was essential to identify the coarsest level of the multiscale features, where a structure was clearly observable, since the analysis of the single wavelets suffers from a loss of geomorphological context. Note that the wavelet transform does not directly analyze structural elements, but the space-frequency atoms composing them. Therefore this identification must be carried out in an indirect way. Even if there 


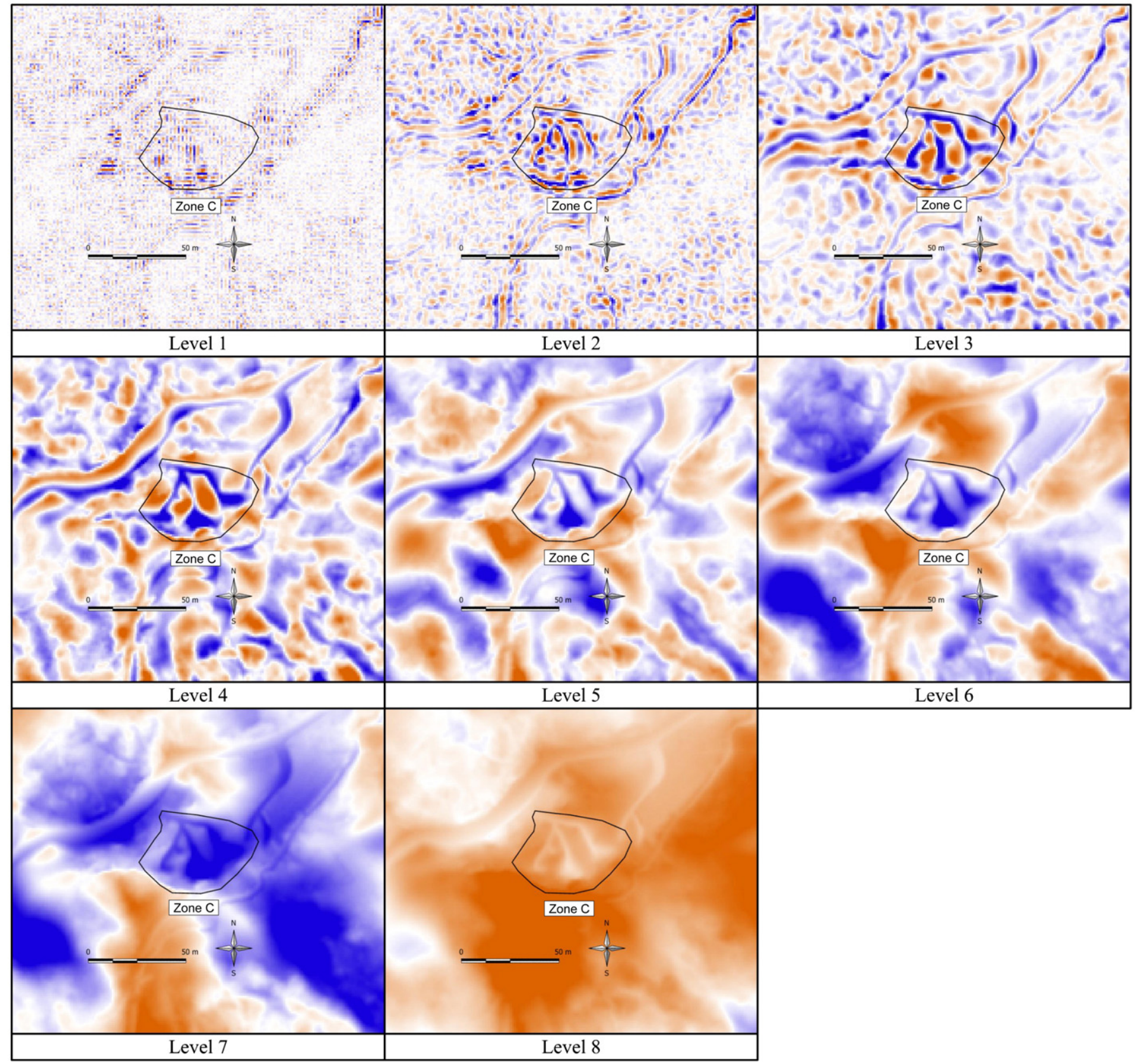

Fig. 10. High-pass results for zone $C$, for decomposition levels 1 to 8 .

is always a relation between the spatial resolution and the spatial size of the structural components of a DEM, the relation between a scale interval, which is linked to a well-defined sub-space of the spatial domain, and the size of a geomorphological structure is not explicit. Highlighting the link between the size of structural components and the spatial domain would provide a good methodology to extract and analyze scale-specific structures. However, as previously stated, we are only able to establish a link between the resolution of the decomposition level and the size of the structure of the landforms represented in the features. Moreover, this link is empirical and emerged only after discussions with the expert.

By means of a visual interpretation of the subband reconstructions (reconstructed high pass coefficients) of the Travers DEM and throughout its global context, we were able to construct empirical geomorphological scale intervals corresponding to the decomposition levels. This only constitutes an indication of the scale interval, at which the different features of a phenomenon can be detected and recognized (Fig. 11). The defined spaces have a local meaning and could lead to a scale-based typology of geomorphological structures.

\section{Conclusion}

Although the use of wavelets is arguably intuitive, only a few researchers of the geomorphological community have used them for the characterization of structural features. This is probably because geomorphometry relies essentially on process-based approaches. In contrast, image analysts are primarily interested in feature detection and characterization to define involved processes.

Even if the wavelet transform does not replace terrain observations, geological mapping, geomorphology and expertise, it provides complementary analytical techniques and methods permitting experts to directly focus on specific topographic elements. However, 


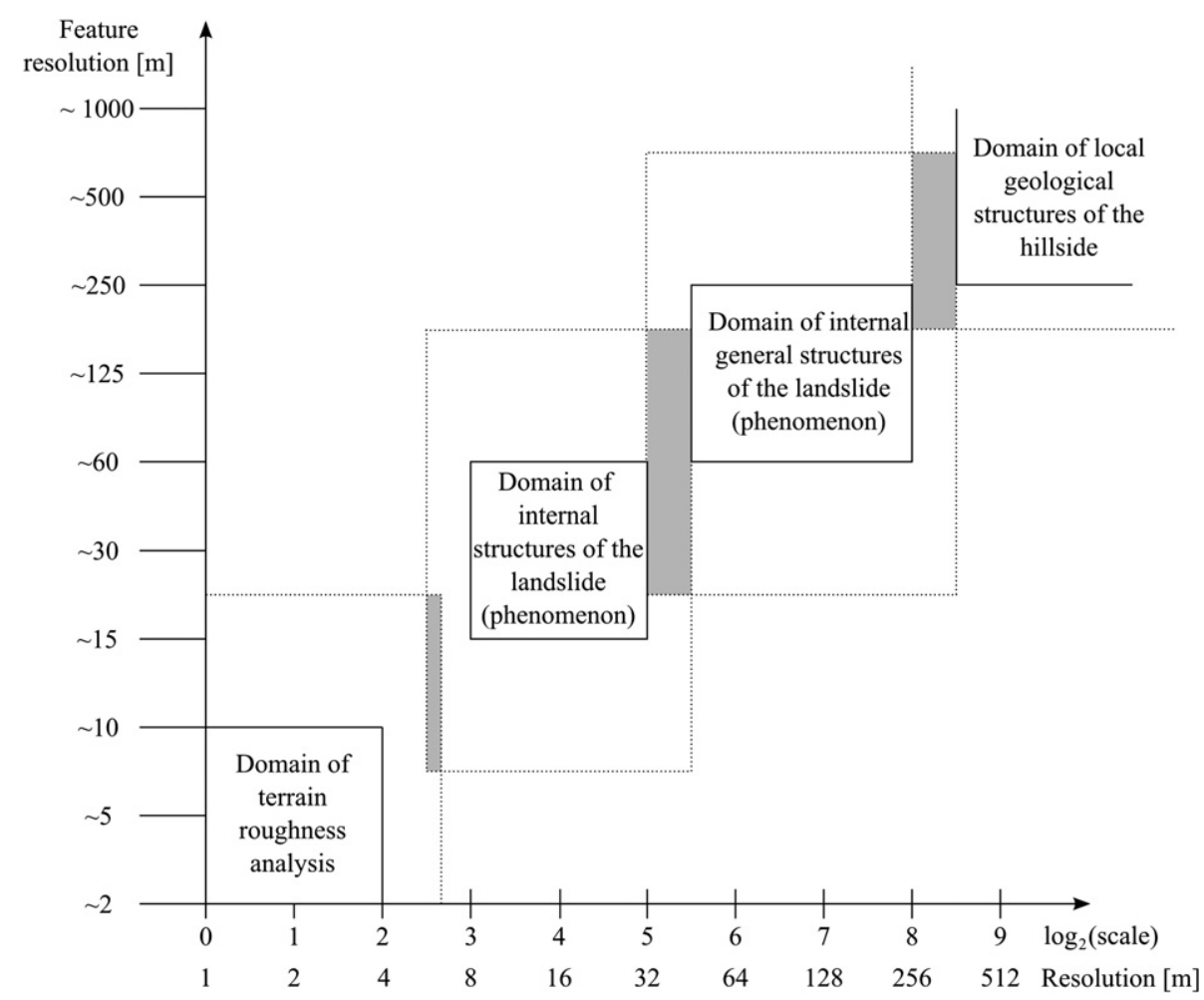

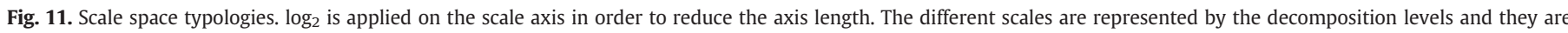
representative of the associated resolution (in $\mathrm{m}$ ) which represents features of specific dimensions (in $\mathrm{m}$ ).

knowledge of the process and of its underlying effects remain necessary because the features extracted from the wavelet decomposition are numerous, and because expert knowledge is still required to choose the best data combinations for each specific application.

Beyond the generalization process, the joint spatial and spectral localization is essential. The capacity to enhance specific spectral information enables users to better understand the topographic structural system at different scales. In our case, the understanding of involved processes and interactions permitted to reach a better understanding of the morphological process, which caused the landslide.

The proposed wavelet coefficients filtering procedure is an innovative and effective analysis technique to support experts in the characterization of a phenomenon and of its topographical aspects. Moreover, this multiscale approach facilitates the analysis by simplifying the complex hierarchy of topographical elements. Although the approach is still exploratory, it makes it now possible to partition the spatial domain using its spectral components and to identify scale-specific features. This new source of information can be efficiently combined with geomorphometric indicators: the first provide knowledge about topographical structure, while the latter brings information regarding the topographical context (Jordan et al., 2005). We firmly believe that the complementarity of image analysis and processing methods with standard geomorphological approaches will be beneficial to geomorphology and geology and usefully enrich them.

\section{Acknowledgments}

The authors would like to thank the Système d'Information du Territoire of the canton of Neuchâtel (SITN, www.sitn.ch) for the support given to this work, and Régis Caloz for initial discussions on the topic. We also acknowledge two anonymous reviewers and Dr. T.
Oguchi for helpful comments on the manuscript. This work was in part supported by the Swiss National Science Foundation under grant PP00P2-123438 (DVDV).

\section{References}

Amgaa, T., 2003. Wavelet-based analysis for object separation from laser altimetry data. Master's thesis, International Institute for Geo-Information Science and Earth Observation, Enschede, Netherlands.

Bjorke, J., Nilsen, S., 2003. Wavelets applied to simplification of digital terrain models. International Journal of Geographical Information Science 17, 601-621.

Blu, T., Unser, M., 2000. The fractional spline wavelet transform: definition and implementation. Proceedings of the Proceedings of the Twenty-Fifth IEEE International Conference on Acoustics, Speech, and Signal Processing (ICASSP'00), I, pp. 512-515.

Creusere, C.D., 2001. Compression of digital elevation maps using nonlinear wavelets. Proceedings of the International Conference on Image Processing ICIP, Thessaloniki, Greece 3, 824-827.

Datcu, M., Luca, D., Seidel, K., 1996. Wavelet-based digital elevation model analysis. Proceedings of the 16th EARSeL Symposium: Integrated Applications for Risk Assessment and Disaster Prevention for the Mediterranean, Malta, pp. 283-290.

De Boer, D.H., 1992. Hierarchies and spatial scale in process geomorphology: a review. Geomorphology 4, 303-318.

Dierkcx, P., 1993. Curve and Surface Fitting with Splines. Oxford Science Publications.

Evans, I.S., 1972. General geomorphometry, derivatives of altitude, and descriptive statistics. In: Chorley, R.J. (Ed.), Spatial Analysis in Geomorphology. Methuen, London, pp. 17-90.

Ferretti, A., Prati, C., Rocca, F., 1999. Multibaseline InSAR DEM reconstruction: the wavelet approach. IEEE Transactions on Geoscience and Remote Sensing 37, 705-715.

Foresti, L., Pozdnoukhov, A., in press. Exploration of alpine orographic precipitation patterns with radar image processing and clustering techniques. Meteorological Applications doi:10.1002/met.272.

Foresti, L., Tuia, D., Kanevski, M., Pozdnoukhov, A., 2011. Learning wind fields with multiple kernels. Stochastic Environmental Research and Risk Assessment 25, 51-66.

Gallant, J.C., Hutchinson, M.F., 1996. Towards an understanding of landscape scale and structure. Proceedings of the Third International Conference/Workshop on Integrating GIS and Environmental Modeling.

Grewe, L., Brooks, R.R., 1997. On localization in the wavelet domain. Proceedings of the 1997 Symposium on Computational Intelligence in Robotics and Automation, pp. 412-418.

Jordan, G., 2007. Digital terrain modelling in GIS environment. In: Peckham, R.J., Jordan, G. (Eds.), Digital Terrain Modelling. Development and Applications in a Policy Support Environment. Series: Lecture Notes in Geoinformation and Cartography. Springer, Berlin, pp. 1-44. 
Jordan, G., Schott, B., 2005. Application of wavelet analysis to the study of spatial pattern of morphotectonic lineaments in digital terrain models. A case study. Remote Sensing of Environment 94, 31-38.

Jordan, G., Meijninger, B.M.L., van Hinsbergen, D.J.J., Meulenkamp, J.E., van Dijk, P.M., 2005. Extraction of morphotectonic features from DEMs: development and applications for study areas in Hungary and NW Greece. International Journal of Applied Earth Observation and Geoinformation 7, 163-182.

Klinkenberg, B., 1992. Fractals and morphometric measures: is there a relationship? Geomorphology 5, 5-20.

Krähenbühl, S., 2007. Prévision de la sensibilité locale d'un versant aux mouvements de terrain par l'analyse des indices topographiques, géologiques et hyrogéologiques Cas du glissement de terrain de Travers (Canton de Neuchâtel, Suisse). Master's thesis, Ecole polytechnique fédérale de Lausanne (EPFL).

Lashermes, B., Foufoula-Georgiou, E., Dietrich, W.E., 2007. Channel network extraction from high resolution topography using wavelets. Geophysical Research Letters 34 .

Lassueur, T., Joost, S., Randin, C.F., 2006. Very high resolution digital elevation models: do they improve models of plant species distribution. Ecological Modelling 198, 139-153.

Mahler, E., 2001. Scale-Dependent Filtering of High Resolution Digital Terrain Models in the Wavelet Domain. Master's thesis, Department of Geography, University of Zurich

Mallat, S., 2000. Une exploration des signaux en ondelettes. Paris: Les éditions de l'école polytechnique.

Marceau, D.J., Hay, G.J., 1999. Remote sensing contributions to the scale issue. Canadian Journal of Remote Sensing 25, 357-366.

Mark, D.M., Aronson, P.B., 1984. Scale-dependent fractal dimensions of topographic surfaces: an empirical investigation, with applications in geomorphology and computer mapping. Mathematical Geology 16, 671-683.
Marr, D., 1982. Vision: A Computational Investigation into the Human Representation and Processing of Visual Information. W. H. Freeman and Company, San Francisco.

Martinoni, D., 2002. Models and Experiments for Quality Handling in Digital Terrain Modeling. Ph.D. thesis, Faculty of Science, University of Zurich.

Ottoson, P., 2001. Compressing digital elevation models with wavelets decomposition. Proceedings of the ScanGIS Conference 2001, As, Norway, pp. 15-31.

Pike, R.J., 2000. Geomorphometry - diversity in quantitative surface analysis. Progress in Physical Geography 24, 1-20.

Schoenberg, I.J., 1946. Contributions to the problem of approximation of equidistant data by analytic functions. Quarterly of Applied Mathematics 4, 45-99.

Unser, M., 1999. Splines, a perfect fit for signal and image processing. IEEE Signal Processing Magazine 16, 22-38.

Unser, M., Blu, T., 1999. Construction of fractional spline wavelet bases. Proceedings of the Proceedings of the SPIE Conference on Mathematical Imaging: Wavelet Applications in Signal and Image Processing, VII, pp. 551-560.

Unser, M., Blu, T., 2003. Wavelet theory demystified. IEEE Transactions on Signal Processing $51,470-483$.

Vetterli, M., 1986. Filter banks allowing perfect reconstruction. Signal Processing 10, 219-244.

Vu, T.T., Tokunaga, M., 2002. Designing of wavelet-based processing system for airborne laser scanner segmentation. Proceedings of the International Workshop on Visualization and Animation Of Landscape, Kunming, China, volume XXX IV.

Wilson, J.P., Gallant, J.C., 2000. Terrain Analysis, Principles and Applications. Wiley, New-York.

Wood, J., 1996. The Geomorphological Characterisation of Digital Elevation. PhD thesis, University of Leicester, UK. 